\title{
Effect of Different Binder Proportions on Properties of Silicon-based Anode Materials
}

\author{
Research Article
}

Volume 2 Issue 2- 2021

\author{
Author Details \\ Chenglong Jiang ${ }^{1}$, Fei $\mathrm{Li}^{1}$, Jing Wang${ }^{2}$, Tianyi Ma ${ }^{1 *}$ \\ ${ }^{1}$ CATARC Automotive Test Center (Tianjin) Co. Ltd., China \\ ${ }^{2}$ School of Materials Science \& Engineering, Beijing Institute of Technology, China
}

${ }^{*}$ Corresponding author

CATARC Automotive Test Center (Tianjin) Co. Ltd., Tianjin 300300, China, School of Materials Science \& Engineering, Beijing Key Laboratory of Environmental Science and Engineering, Beijing Institute of Technology, Beijing 100081, China

Article History

Received: May 17, 2021 Accepted: May 19, 2021 Published: May 20, 2021

\begin{abstract}
With the development of lithium-ion battery technology, silicon anode is deemed as an ideal next-generation anode materials by virtue of its extremely high specific capacity. Nevertheless, higher requirements are raised for the properties of the binder owing to the high volume change rate of silicon anode during charging and discharging and the thickening of SEI film. In respect of the binders such as polyacrylic acid (PAA), polyvinyl alcohol (PVA), sodium alginate (Alginate), sodium carboxymethylcellulose (CMC) in possession of large-scale application value, this paper studies the effects of different binders and mixed binders with different ratios on the properties of silicon anode materials. As a result, it is suggested that the binders acquired when PAA:PVA=9:1 can render it possible for the electrode to be provided with better charge-discharge cyclic performance.
\end{abstract}

Keywords: Lithium-ion battery; Silicon anode; Binder

\section{Introduction}

With regard to the key factor influencing the development of the properties of lithium batteries, it is attributable to the development of anode and cathode materials [1-3]. On the anode side, traditional graphite electrodes will produce no volume effect during charge and discharge, and the properties of binders exert no significant influence on the charge-discharge cyclic performance [4-6]. With the development of high-capacity silicon materials, silicon-based materials have aroused more and more concern and a large amount of research has been carried out, but there is a huge volume effect in silicon materials [7-9]. Thus, silicon-based materials raise comparatively high requirements for binders; with an intent to gain stable cyclic performance of silicon materials, it is requisite to explore and study silicon-based binders $[10,11]$.

In view of the fact that traditional binders, such as PVDF, can no longer be in line with the application requirements of silicon-based materials. To address this problem, upholding the concept of green environmental protection and abandoning the dependence on the binder of organic solvent system, this experiment chiefly probes into water-based binders; NanoSi and graphite are taken as active materials, and acetylene black is regarded as conductive agent. With reference to previous literature research, water-based binders studied by researchers currently include polyacrylic acid (PAA), polyvinyl alcohol (PVA), sodium alginate (Alginate), sodium carboxymethylcellulose (CMC), guar gum, xanthan gum, etc.; in comparison with the traditional binder PVDF, the cyclic performance of these binders is enhanced to varying degrees [12-14]. Enlightened by previous literature, the abovementioned binders will be used alone in this paper, or two kinds of binders with different properties and different functional groups are mixed in a certain proportion as a new composite binder and mixed with active materials for coating, and then the effects of different binders on electrode performance are compared, with a view to find a binder that can effectively alleviate the volume effect of silicon-based materials and enhance their cyclic performance.

\section{Results and Discussions}

With NanoSi/graphite as active material and acetylene black as conductive agent, in this experiment, it is required to study the effect of different binders on the cyclic performance of active materials when such binders are mixed with the above-mentioned materials respectively. The material composition in the electrode is stated 
below: nano Si/graphite active material: conductive agent of acetylene black: binder $=7: 1.5: 1.5$, and the particle size of nano $\mathrm{Si}$ is less than $200 \mathrm{~nm}$; therein, in the nano Si/graphite active materials, the mass ratio of nano-Si and graphite is $28.57 \%$ and $71.43 \%$, respectively. The binders used in the experiment are polyacrylic acid (PAA), polyacrylic acid and polyvinyl alcohol composite binder: polyacrylic acid (PAA): polyvinyl alcohol (PVA)=9:1, PAA:PVA=8:2, PAA:PVA=7:3, PAA:PVA=6:4 (weight ratio), sodium alginate (Alginate), sodium carboxymethylcellulose (CMC), PVDF.

Based on the above ratio, weigh all kinds of materials, grind, mix and coat them. The vacuum drying process of the coating with the composite binder of polyacrylic acid and polyvinyl alcohol is divided into two stages: dry it at $100^{\circ} \mathrm{C}$ for $5 \mathrm{~h}$ and then dry it at $150^{\circ} \mathrm{C}$ for $1 \mathrm{~h}$, so that the carboxyl groups of polyacrylic acid and hydroxyl groups of polyvinyl alcohol react to form ester bonds. Assemble all types of pole pieces into batteries to test all types of electrochemical properties, and compare the effects of different binders on the cyclic performance of silicon-based materials, as presented in Figure 1.

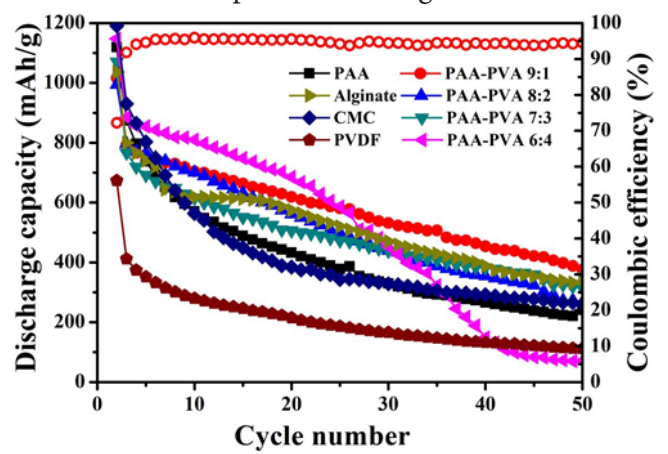

Figure 1: Comparison of cyclic performance of different binder with NanoSi/graphite as active material.
Figure 1 is a comparison chart of cyclic performance of different binders with nano-Si/graphite as active material. Table 1 indicates reversible capacity and first coulombic efficiency of different binders with nano-Si/graphite as active materials. As the content of $\mathrm{Si}$ in nano-Si/graphite active materials is $28.57 \%$, and the proportion of graphite is $71.43 \%$, the theoretical capacity of the composite material is calculated to be $1467.1 \mathrm{mAh} / \mathrm{g}$. Figure 1 indicates that when PVDF is used as a binder in combination with nano-Si/graphite active materials, the cyclic performance is distinctly inferior to other waterbased binders, and the capacity is only $111.9 \mathrm{mAh} / \mathrm{g}$ after 50 cycles, while the binding performance of pure PAA as a binder is similar to CMC; when PAA and PVA are compounded in a certain proportion, the cycle performance of the electrode will be obviously enhanced, because they will be combined with each other by ester bond after being compounded at a certain temperature, and the hydroxyl group of polyvinyl alcohol will form hydrogen bond with the surface of nano-silicon, so that the binder and nano-Si will be firmly combined; therein, when the ratio of the composite binder PAA-PVA is 8:2 and $7: 3$, the bonding capacity is similar to that of sodium alginate (Alg). When the ratio of PVA in the composite binder PAA-PVA is further increased, that is, PAA:PVA=6:4, although the electrode shows good cyclic performance before 20 cycles, the subsequent capacity will decrease sharply; this is because PVA itself is brittle, and when the proportion of PVA in the composite binder is too high, it will reduce the flexibility of the composite binder, make the binder lose its capability to buffer the volume expansion of silicon, and shorten the cycle life of the electrode. In comparison, when PAA:PVA=9:1, the composite binder boasts the best cyclic performance, and the capacity is $365.1 \mathrm{mAh} / \mathrm{g}$ after 50 cycles of charge and discharge. At this ratio, the composite binder is characterized by the best flexibility and viscosity, and the binder network formed by PAA and PVA through ester bond can form the best contact state with nano-Si.

Table 1: The reversible capacity and initial coulombic efficiency of different binder with NanoSi/graphite as active material.

\begin{tabular}{|c|c|c|c|c|c|c|c|c|}
\hline & PAA & $\mathbf{0 9 : 0 1}$ & $\mathbf{0 8 : 0 2}$ & $\mathbf{0 7 : 0 3}$ & $\mathbf{0 6 : 0 4}$ & Alg & CMC & PVDF \\
\hline $1^{\text {st }}$ cycle $(\mathrm{mAh} / \mathrm{g})$ & 1119.3 & 1016.5 & 994.1 & 1070.3 & 1147.2 & 1036.5 & 1190.4 & 673.9 \\
\hline $50^{\text {th }}$ cycle $(\mathrm{mAh} / \mathrm{g})$ & 241.2 & 375.9 & 265.8 & 313.3 & 71 & 345.6 & 261.3 & 111.9 \\
\hline Initial coulombic efficiency $(\%)$ & 74.4 & 72.2 & 74.5 & 66.4 & 71.8 & 72 & 74 & 63.5 \\
\hline
\end{tabular}

Figure 2 is the charge and discharge curve of different binders with nano-Si/graphite as active material in the first three cycles, Figure $2 \mathrm{a}$ is a charge-discharge curve of PVDF as binder for the first three cycles, and the binder used in Figure $3 b$ is at a ratio of PAA:PVA=9:1. It can be learned from Figure 2a that a comparatively long voltage platform appears around $0.5 \mathrm{~V}$ during the first discharge cycle, and this voltage platform disappears in the subsequent cycle, thus corresponding to the formation of SEI film on the material surface; Figure $2 b$ reveals that the discharge voltage platform appears at about $0.2 \mathrm{~V}$ in the first cycle, and this platform disappears in the subsequent cycle, which also corresponds to the formation of SEI film on the material surface. It can be observed from the figure that the charge capacity undergoes obvious changes in the first three cycles when PVDF is taken as binder, and the charge capacity does not change basically in the first three cycles when PAA:PVA=9:1 is used as binder, which suggests that PAA:PVA=9:1 can make the electrode provided with stable electrochemical performance.

Figure 3 is cyclic voltammetry $(\mathrm{CV})$ curve of nano-Si/graphite as active material with different binders in use. Figure 3a shows a weak reduction peak at about $1.29 \mathrm{~V}$ and a comparatively wide reduction peak at about $0.54 \mathrm{~V}$ during cathode scanning with binder PVDF from $2.74 \mathrm{~V}\left(\mathrm{vs} . \mathrm{Li} / \mathrm{Li}^{+}\right)$to $0 \mathrm{~V}$ in the first cycle. The reduction peaks at $1.29 \mathrm{~V}$ and $0.54 \mathrm{~V}$ disappear during the subsequent cycle, corresponding to the formation of SEI film on the surface of the material while the oxidation peak appears at about $0.37 \mathrm{~V}$, which corresponds to the lithium removal process of active materials. In Figure 3b, binder is used with PAA:PVA=9:1, and the reduction peaks appear at about $0.66 \mathrm{~V}$ and $0.17 \mathrm{~V}$ during the cathodic scanning from $2.0 \mathrm{~V}\left(\mathrm{vs} \cdot \mathrm{Li} / \mathrm{Li}^{+}\right)$to $0 \mathrm{~V}$ in the first cycle, and the two peaks disappear during the subsequent cycle, corresponding to the formation process of SEI film of solid electrolyte. Oxidation peaks at about $0.30 \mathrm{~V}$ and $0.50 \mathrm{~V}$ correspond to the lithium removal process of active materials.

Figure 4 is the AC impedance spectrum of nano-Si/graphite as active materials using different binders. The cell impedance $\left(\mathrm{R}_{\text {cell }}\right)$ of Li-ion battery includes electrolyte impedance $\left(\mathrm{R}_{\mathrm{s}}\right)$, charge transfer impedance $\left(\mathrm{R}_{\mathrm{ct}}\right.$ or electrochemical reaction impedance) at the interface between electrode and electrolyte, and diffusion Warburg impedance $\left(\mathrm{Z}_{\mathrm{w}}\right)$ of lithium ions near the interface between electrode and electrolyte. Wherein, the reaction at the interface of Li-ion battery will incur the increased battery impedance and the decreased capacity of anode materials. It can be learned from Figure 4 that the impedance spectrum of the composite electrode is made up of a semicircle in the high frequency zone and a diagonal line in the low frequency zone. To be specific, the intersection of impedance spectrum curve and $Z^{\prime}$ axis in high frequency zone is $R_{s}$, the semicircle in high frequency zone stands for $\mathrm{R}_{\mathrm{ct}}$, and the diagonal line in low frequency zone corresponds to $\mathrm{Z}_{\mathrm{w}}$. The $\mathrm{R}_{\mathrm{ct}}$ of PVDF with nano-Si/graphite as active materials and PAA-PVA 9:1 as binder electrode is $38.6 \Omega$ and $44.0 \Omega$ respectively. Thus, different binders exert no distinct impacts on the charge transfer impedance. 

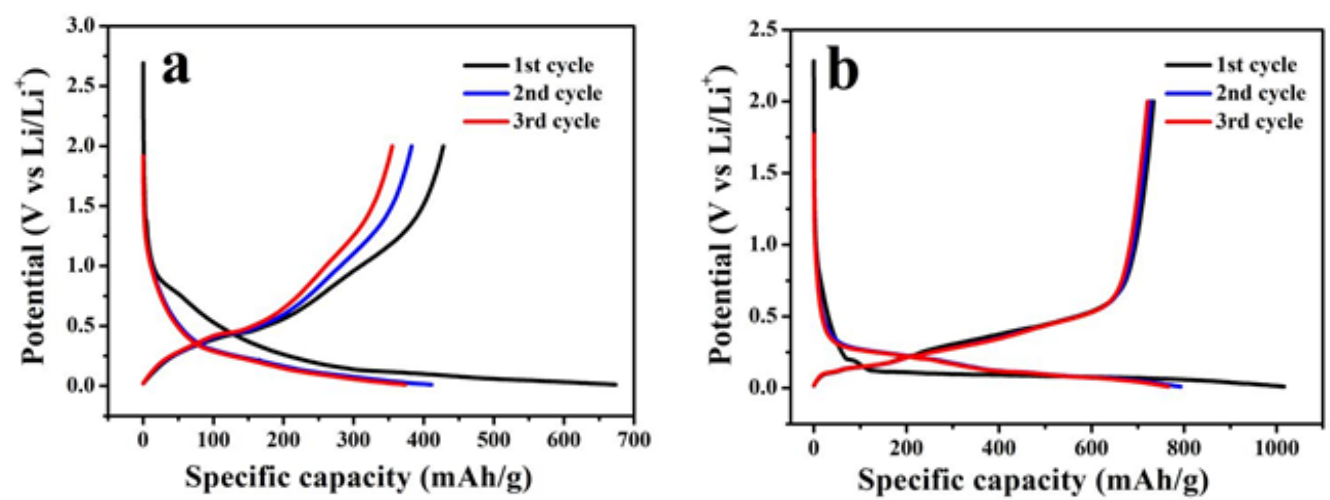

Figure 2: The first three charge-discharge curves of binder(a)PVDF (b)PAA-PVA 9:1 with NanoSi/ graphite as active material.
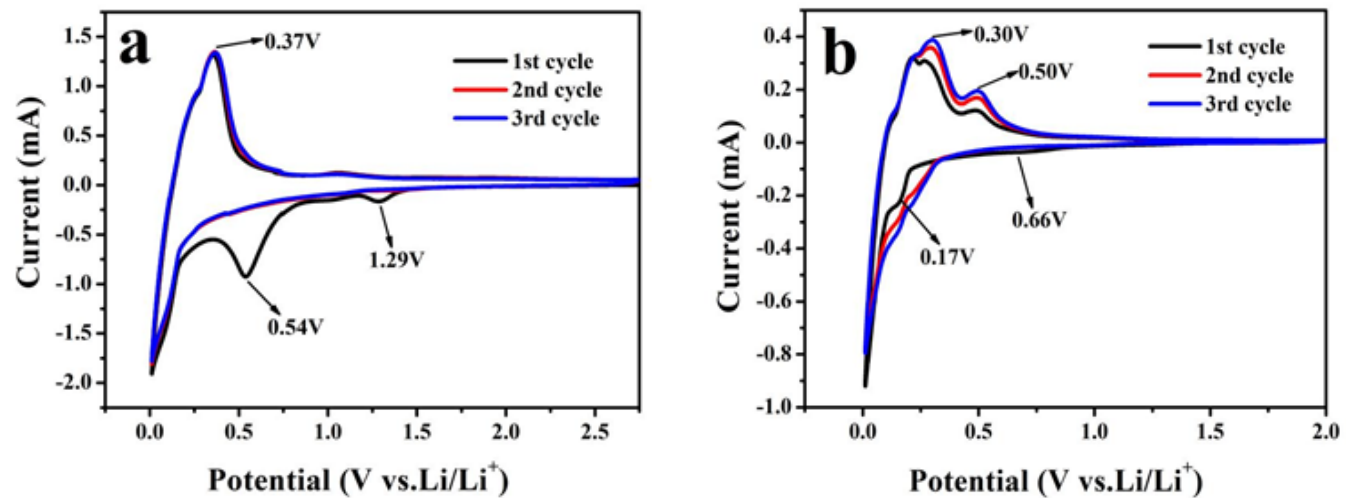

Figure 3: CV curves of binder (a)PVDF (b)PAA-PVA 9:1 with NanoSi/ graphite as active material.

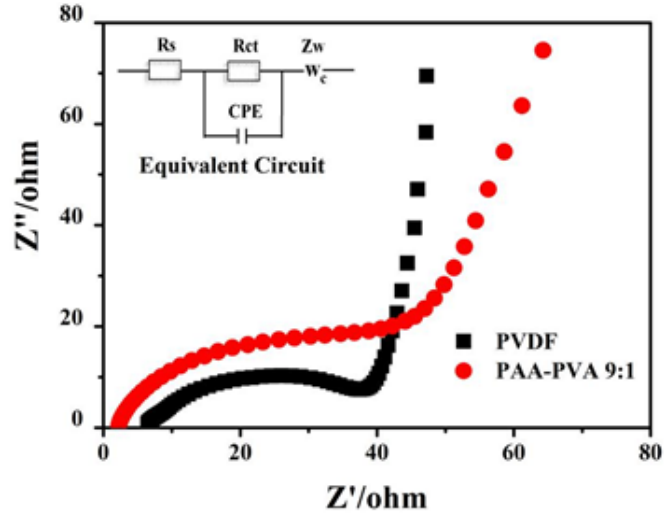

Figure 4: Typical Nyquist plots of binder (a)PVDF (b)PAA-PVA 9:1 with NanoSi/graphite as active material.

\section{Conclusion}

Silicon materials are characterized by both high specific capacity and enormous volume effect simultaneous. With an intent to cater to these characteristics of silicon materials, this chapter probes into the binders suitable for silicon-based materials, and also studies water-based binders in the concept of green environmental protection.

Taking nano-Si/graphite as active materials, the researcher compares the cyclic performance of different binders. The binders used in the experiment are polyacrylic acid (PAA), polyacrylic acid and polyvinyl alcohol composite binder: polyacrylic acid (PAA):polyvinyl alcohol $(\mathrm{PVA})=9: 1$, PAA:PVA=8:2, PAA:PVA=7:3, PAA:PVA=6:4 (weight ratio), sodium alginate (Alginate), sodium carboxymethylcellulose (CMC), PVDF. The above binders are respectively mixed with active materials for coating; and by comparison, it can be learned that when PAA:PVA=9:1, the binders acquired by means of compounding can enable the electrode to be provided with the best charge-discharge cyclic performance. Hence, the sample used under the condition that
PAA:PVA=9:1 is expected to become a binder suitable for siliconbased materials on the strength of further research and improvement.

\section{References}

1. M Li, J Lu, Z Chen, Khalil Amine (2018) 30 years of lithium-ion batteries. Adv Mater 30: 1800561.

2. N Nitta, F Wu, J T Lee, Gleb Yushin (2015) Li-ion battery materials: present and future. Materials today 18(5): 252-264.

3. H Hesse, M Schimpe, D Kucevic, A Jossen (2017) Lithium-ion battery storage for the grid-A review of stationary battery storage system design tailored for applications in modern power grids. Energies 10(12): 2107.

4. ZX Xu, J Yang, HP Li, Y Nuli, J Wang (2019) Electrolytes for advanced lithium ion batteries using silicon-based anodes. Journal of Materials Chemistry A 7: 9432-9446.

5. YG Zhang, N Du, DR Yang (2019) Designing superior solid electrolyte interfaces on silicon anodes for high-performance lithium-ion batteries. Nanoscale 11(41): 19086-19104.

6. Y Tan, K Wang (2019) Silicon-based Anode Materials Applied in High Specific Energy Lithium-ion Batteries: a Review. Journal of Inorganic Materials 34(4): 349-357.

7. B Zhu, X Wang, P Yao, J Li, J Zhu (2019) Towards the high energy density lithium battery anodes: Silicon and Lithium. Chem Sci 10: 7132-7148.

8. T Ma, H Xu, X Yu, H Li, W Zhang, et al. (2019) Lithiation Behavior of Coaxial Hollow Nanocables of Carbon-Silicon Composite. ACS Nano 13(2): 2274-2280.

9. Q Ji, Q Ji, Y Xia, J Zhu, B Hu, et al. (2019) Practically Relevant Research on Silicon-Based Lithium-Ion Battery Anodes. Advanced Battery Materials pp. 261-305.

10. J Liu, Q Duan, M Ma, C Zhao, J Sun, et al. (2020) Aging mechanisms and thermal stability of aged commercial 18650 lithium ion battery induced by slight overcharging cycling. Journal of Power Sources 445: 227263. 
11. X Han, L Lu, Y Zheng, X Feng, Z Li, et al. (2019) A review on the key issues of the lithium ion battery degradation among the whole life cycle. eTransportation 1: 100005

12. K Brandt, J Garche (2019) General Overview of Li-Secondary Battery Safety Issues, in: Electrochemical Power Sources: Fundamentals, Systems, and Applications, Elsevier; pp. 127-141.
13. P Zhang, J Zhu, M Wang, N Imanishi, O Yamamoto (2018) Lithium dendrite suppression and cycling efficiency of lithium anode. Electrochemistry Communications 87: 27-30.

14. Q Pang, X Liang, I R Kochetkov, P Hartmann, L F Nazar (2018) Stabilizing lithium plating by a biphasic surface layer formed in situ. Angewandte Chemie International Edition 57(31): 9795-9798. 